\title{
UNIQUENESS OF BOUNDED SOLUTIONS FOR THE HOMOGENEOUS LANDAU EQUATION WITH A COULOMB POTENTIAL
}

\author{
NICOLAS FOURNIER
}

\begin{abstract}
We prove the uniqueness of bounded solutions for the spatially homogeneous FokkerPlanck-Landau equation with a Coulomb potential. Since the local (in time) existence of such solutions has been proved by Arsen'ev-Peskov [2, we deduce a local well-posedness result. The stability with respect to the initial condition is also checked.
\end{abstract}

\section{INTRODUCTION}

We consider the spatially homogeneous Landau equation for a Coulomb potential. This equation of kinetic physics, also called Fokker-Planck-Landau equation, has been derived from the Boltzmann equation by Landau. It describes the density $f_{t}(v)$ of particles with velocity $v \in \mathbb{R}^{3}$ at time $t \geq 0$ in a spatially homogeneous dilute plasma:

$$
\partial_{t} f_{t}(v)=\frac{1}{2} \sum_{i, j=1}^{3} \partial_{i}\left(\int_{\mathbb{R}^{3}} a_{i j}\left(v-v^{*}\right)\left[f_{t}\left(v^{*}\right) \partial_{j} f_{t}(v)-f_{t}(v) \partial_{j}^{*} f_{t}\left(v^{*}\right)\right] d v^{*}\right) .
$$

Here $\partial_{t}=\frac{\partial}{\partial t}, \partial_{i}=\frac{\partial}{\partial v_{i}}, \partial_{i}^{*}=\frac{\partial}{\partial v_{i}^{*}}$ and for $z \in \mathbb{R}^{3}, a(z)$ is the symmetric nonnegative matrix

$$
a_{i j}(z)=|z|^{-3}\left(|z|^{2} \delta_{i j}-z_{i} z_{j}\right) .
$$

We refer to Villani [14, 15, Alexandre-Villani [1] and the references therein for many information on this equation, which has been widely used in plasma physics. Let us mention that conservations of mass, momentum and kinetic energy hold a priori, that is for $t \geq 0, \int \varphi(v) f_{t}(v) d v=$ $\int \varphi(v) f_{0}(v) d v$, for $\varphi(v)=1, v,|v|^{2}$. We classically may assume without loss of generality that $\int f_{0}(v) d v=1$. Another fundamental estimate, that we will not use here, is the decay of entropy: $\int f_{t}(v) \log f_{t}(v) d v \leq \int f_{0}(v) \log f_{0}(v) d v$ for all $t \geq 0$.

Assume that in a dilute gas or plasma, particles collide by pairs, due to a repulsive force proportional to $1 / r^{s}$, where $r$ stands for the distance between the two particles. Then if $s \in(2, \infty)$, the velocity distribution solves the corresponding Boltzmann equation [14, 15. But if $s=2$, the Boltzmann equation is meaningless [14] and is often replaced by the Landau equation (11). However, there are also many mathematical works on the Landau equation where $|z|^{-3}$ is replaced by $|z|^{\gamma}$ in (2), with $\gamma=(s-5) /(s-1) \in[-3,1)$. One usually speaks of hard potentials when $\gamma \in(0,1)$ (i.e. $s>5$ ), Maxwell molecules when $\gamma=0$ (i.e. $s=5$ ), soft potentials when $\gamma \in(-3,0)$ (i.e. $s \in(2,5)$ ), Coulomb potential when $\gamma=-3$ (i.e. $s=2$ ).

When $\gamma>-3$, the Landau equation can be seen as an approximation of the corresponding Boltzmann equation in the asymptotic of grazing collisions [14. This can help to understand the effect of grazing collisions in the Boltzmann equation without cutoff and is also of interest

2000 Mathematics Subject Classification. 82C40.

Key words and phrases. Kinetic equations, Plasma physics, Fokker-Planck-Landau equation, Coulomb potential. 
numerically. But it seems that only the Landau equation with a Coulomb potential has considerable importance in plasma physics.

The existence theory for the homogeneous Landau equation is quite complete. In [14, Villani has proved the global existence of weak solutions to the homogeneous Landau equation for all possible potentials $\gamma \in[-3,1)$, for any initial condition with finite mass, energy and entropy. He also showed in this paper that the solution to the Landau equation can be seen as the limit of a sequence of solutions to some suitable Boltzmann equations. It is worth noting that for $\gamma \in[-2,1)$, the tools used in 14 are quite classical. But for $\gamma \in[-3,-2)$, Villani uses some very fine a priori estimates provided by the entropy dissipation. The paper of Alexandre-Villani [1] contains some existence results in the much more difficult inhomogeneous case.

Uniqueness for the Landau equation is much less well-understood, even in the spatially homogeneous case. To our knowledge, this problem is still completely open in the realistic Coulomb case. Of course, uniqueness is very important, even from the physical point of view: if uniqueness is not holding, this means that the equation is not well-posed and thus that some additional physical conditions have to be added.

Let us summarize the situations in which uniqueness for the homogeneous Landau equation is known to hold. The initial condition $f_{0}$ is always supposed to have finite mass and energy, $\int f_{0}(v)\left(1+|v|^{2}\right) d v<\infty$. On has global uniqueness when $\gamma=0$, see Villani [13, when $\gamma \in(0,1)$ and $\left.\int f_{0}^{2}(v)\left(1+|v|^{q}\right)\right] d v<\infty$ for some $q>5 \gamma+15$, see Desvillettes-Villani [5], and when $\gamma \in(-2,0$ ] and $\int\left[f_{0}(v)|v|^{q}+f_{0}(v) \log f_{0}(v)\right] d v<\infty$ for some $q>\gamma^{2} /(2+\gamma)$, see [8]. One has local (in time) uniqueness when $\gamma \in(-3,-2]$ and $f_{0} \in L^{p}$ for some $p>3 /(3+\gamma)$, see [8].

The goal of this paper is to extend this final result to the case of a Coulomb potential $\gamma=-3$, showing local uniqueness for bounded initial conditions with finite mass and energy. As a matter of fact, we will show that for any $T$, uniqueness holds in the space $L^{1}\left([0, T], L^{\infty}\left(\mathbb{R}^{3}\right)\right)$. But the only known result that provides existence of such solutions is that of Arsen'ev-Peskov [2] for $f_{0}$ bounded, one can find $T_{*}\left(f_{0}\right)>0$ and a solution to (1) lying to $L^{\infty}\left(\left[0, T_{*}\left(f_{0}\right)\right] \times \mathbb{R}^{3}\right)$. Thus our uniqueness result is not so satisfying at the moment, since it concerns a functional space in which solutions are known to belong only for bounded time interval.

The Coulomb case is really more difficult than the case $\gamma>-3$, essentially because $|z|^{-3}$ is not integrable near 0 . Thus while the global scheme of the proof is the same as in [8], the central computations are much more delicate and borderline. It seems that Villani's existence results can be extended to the case $\gamma \in(-4,-3]$, see [14, p 284], but we are here really at the boundary of our possibilities.

Let us finally mention that the starting point of our proof is the famous work of Tanaka 12, who proved the first uniqueness result for the Boltzmann equation without cutoff (for Maxwell molecules). We already used Tanaka's approach to study uniqueness for the Boltzmann equation without cutoff for hard and soft potentials [6, 7].

\section{MAIN RESULT}

Let $\mathcal{P}$ be the set of probability measures on $\mathbb{R}^{3}$ and

$$
\mathcal{P}_{2}=\left\{f \in \mathcal{P}, m_{2}(f)<\infty\right\}, \quad \text { where } \quad m_{2}(f)=\int_{\mathbb{R}^{3}}|v|^{2} f(d v) .
$$

For a measurable family $\left(f_{t}\right)_{t \in[0, T]} \subset \mathcal{P}$, we say that

$$
\left(f_{t}\right)_{t \in[0, T]} \in L^{\infty}\left([0, T], \mathcal{P}_{2}\right) \quad \text { if } \quad \sup _{[0, T]} m_{2}\left(f_{t}\right)<\infty .
$$

Observe that any reasonable solution to (11) belongs to $L^{\infty}\left([0, T], \mathcal{P}_{2}\right)$, because $m_{2}\left(f_{t}\right)=m_{2}\left(f_{0}\right)$. 
When $f \in \mathcal{P}$ has a bounded density, we say that $f \in L^{\infty}$, we also denote by $f$ its density and by $\|f\|_{\infty}$ its $L^{\infty}$-norm. For a measurable family $\left(f_{t}\right)_{t \in[0, T]} \subset \mathcal{P}$, we say that

$$
\left(f_{t}\right)_{t \in[0, T]} \in L^{1}\left([0, T], L^{\infty}\right) \quad \text { if } \quad \int_{0}^{T}\left\|f_{t}\right\|_{\infty} d t<\infty .
$$

We denote by $C_{b}^{2}$ the set of $C^{2}$ functions $\varphi: \mathbb{R}^{3} \mapsto \mathbb{R}$ with bounded derivatives of order 0 to 2 . For $\varphi \in C_{b}^{2}$ and $v, v^{*} \in \mathbb{R}^{3}$, we introduce

$$
\begin{aligned}
& L \varphi\left(v, v^{*}\right)=\frac{1}{2} \sum_{i, j=1}^{3} a_{i j}\left(v-v^{*}\right) \partial_{i j}^{2} \varphi(v)+\sum_{i=1}^{3} b_{i}\left(v-v^{*}\right) \partial_{i} \varphi(v) \\
& \text { where } \quad b_{i}(z)=\sum_{j=1}^{3} \partial_{j} a_{i j}(z)=-2|z|^{-3} z_{i} .
\end{aligned}
$$

Definition 1. We say that $\left(f_{t}\right)_{t \in[0, T]}$ is a weak solution to (11) starting from $f_{0} \in \mathcal{P}_{2}$ if $\left(f_{t}\right)_{t \in[0, T]} \in$ $L^{\infty}\left([0, T], \mathcal{P}_{2}\right) \cap L^{1}\left([0, T], L^{\infty}\right)$ and if for any $\varphi \in \mathcal{C}_{b}^{2}$, any $t \in[0, T]$,

$$
\int_{\mathbb{R}^{3}} \varphi(v) f_{t}(v) d v=\int_{\mathbb{R}^{3}} \varphi(v) f_{0}(v) d v+\int_{0}^{t} \int_{\mathbb{R}^{3}} \int_{\mathbb{R}^{3}} f_{t}(v) f_{t}\left(v^{*}\right) L \varphi\left(v, v^{*}\right) d v d v^{*} d s .
$$

For $\varphi \in C_{b}^{2}$, one has $\left|L \varphi\left(v, v^{*}\right)\right| \leq C_{\varphi}\left(\left|v-v^{*}\right|^{-1}+\left|v-v^{*}\right|^{-2}\right)$ for some constant $C_{\varphi}$. Thus (8) and our conditions on $\left(f_{t}\right)_{t \in[0, T]}$ ensure us that all the terms are well-defined in (5). The weak formulation (5) is standard and can be found in [14, Eq. (36)].

We will widely use the Wasserstein distance $\mathcal{W}_{2}$, defined for $f, \tilde{f} \in \mathcal{P}_{2}$, by

$$
\begin{aligned}
\mathcal{W}_{2}^{2}(f, \tilde{f}) & =\inf \left\{\mathbb{E}\left[|V-\tilde{V}|^{2}\right], V \sim f, \tilde{V} \sim \tilde{f}\right\} \\
& =\inf \left\{\int_{\mathbb{R}^{3} \times \mathbb{R}^{3}}|v-\tilde{v}|^{2} R(d v, d \tilde{v}), R \in \mathcal{H}(f, \tilde{f})\right\} .
\end{aligned}
$$

Here $V \sim f$ means that $V$ is a $\mathbb{R}^{3}$-valued random variable with law $f$ and $\mathcal{H}(f, \tilde{f})$ is the set of all probability measures on $\mathbb{R}^{3} \times \mathbb{R}^{3}$ with marginals $f$ and $\tilde{f}$. The set $\left(\mathcal{P}_{2}, \mathcal{W}_{2}\right)$ is a Polish space and its topology is slightly stronger than the weak topology, see Villani [16, Theorem 7.12]. It is well-known [16, Chapter 1] that the infimum is reached: for $f, \tilde{f} \in \mathcal{P}_{2}$, we can find $R \in \mathcal{H}(f, \tilde{f})$ and $V \sim f, \tilde{V} \sim f$ such that $\mathcal{W}_{2}^{2}(f, \tilde{f})=\int_{\mathbb{R}^{3} \times \mathbb{R}^{3}}|v-\tilde{v}|^{2} R(d v, d \tilde{v})=\mathbb{E}\left[|V-\tilde{V}|^{2}\right]$.

Our main result reads as follows.

Theorem 2. Let $T>0$.

(i) For $f_{0} \in \mathcal{P}_{2}$, there is at most one weak solution to (1) starting from $f_{0}$ and belonging to $L^{\infty}\left([0, T], \mathcal{P}_{2}\right) \cap L^{1}\left([0, T], L^{\infty}\right)$.

(ii) Assume that we have some weak solutions $\left(f_{t}\right)_{t \in[0, T]}$ and $\left(f_{t}^{n}\right)_{t \in[0, T]}$ to (11), all belonging to $L^{\infty}\left([0, T], \mathcal{P}_{2}\right) \cap L^{1}\left([0, T], L^{\infty}\right)$. If $\sup _{n} \int_{0}^{T}\left\|f_{t}^{n}\right\|_{\infty} d t<\infty$ and $\lim _{n} \mathcal{W}_{2}\left(f_{0}^{n}, f_{0}\right)=0$, then $\lim _{n} \sup _{[0, T]} \mathcal{W}_{2}\left(f_{t}^{n}, f_{t}\right)=0$

Arsen'ev-Peskov [2] have proved the following existence result. Let $A>0$ be fixed. There exist some constants $T_{A}>0$ and $C_{A}$ depending only on $A$ (with $\lim _{A \backslash 0} T_{A}=+\infty$ ) such that for any $f_{0} \in L^{\infty} \cap \mathcal{P}_{2}$ with $\left\|f_{0}\right\|_{\infty} \leq A$, there exists a weak solution $\left(f_{t}\right)_{t \in\left[0, T_{A}\right]}$ to (11) satisfying $\sup _{\left[0, T_{A}\right]}\left\|f_{t}\right\|_{\infty} \leq C_{A}$. Theorem 2 ensures us that this solution is unique and continuous with respect to the initial condition. The result in [2] is based on the (formally easy) estimate $\frac{d}{d t}\left\|f_{t}\right\|_{\infty} \leq$ $C\left(1+\left\|f_{t}\right\|_{\infty}^{2}\right)$. 
In the next section, we establish some fundamental regularity estimates on the coefficients $a$ and $b$ of the Landau equation and we recall a well-known generalization of the Gronwall Lemma. The proof of Theorem 2 is handled in Sections 4 and 5 . In the whole paper, $C$ stands for a universal constant, whose value changes from line to line.

\section{Preliminaries}

We will study the Landau equation through a stochastic differential equation whose coefficients are $b$ (recall (4)) and $\sigma$, defined for $z \in \mathbb{R}^{3}$ by

$$
\sigma(z)=|z|^{\frac{-3}{2}}\left(\begin{array}{ccc}
z_{2} & -z_{3} & 0 \\
-z_{1} & 0 & z_{3} \\
0 & z_{1} & -z_{2}
\end{array}\right) .
$$

For all $z \in \mathbb{R}^{3}$, one has $\sigma(z) \cdot(\sigma(z))^{t}=a(z)$, recall (2).

Lemma 3. For any $z, \tilde{z} \in \mathbb{R}^{3}$,

$$
\begin{aligned}
|\sigma(z)-\sigma(\tilde{z})|^{2} & \leq C \min \left\{|z-\tilde{z}|^{2}\left(|z|^{-3}+|\tilde{z}|^{-3}\right) ;|z|^{-1}+|\tilde{z}|^{-1}\right\} \\
|b(z)-b(\tilde{z})| & \leq C \min \left\{|z-\tilde{z}|\left(|z|^{-3}+|\tilde{z}|^{-3}\right) ;|z|^{-2}+|\tilde{z}|^{-2}\right\} .
\end{aligned}
$$

Proof. First, we have $|\sigma(z)| \leq|z|^{-1 / 2}$, whence $|\sigma(z)-\sigma(\tilde{z})|^{2} \leq 2\left(|z|^{-1}+|\tilde{z}|^{-1}\right)$. Next,

$$
\begin{aligned}
|\sigma(z)-\sigma(\tilde{z})| & \leq\left.|| z\right|^{-3 / 2}-|\tilde{z}|^{-3 / 2}|\cdot| z|+| z-\left.\tilde{z}|\cdot| \tilde{z}\right|^{-3 / 2} \\
& \leq \frac{3}{2}|z| \cdot|z-\tilde{z}| \max \left(|z|^{-5 / 2},|\tilde{z}|^{-5 / 2}\right)+|z-\tilde{z}|\left(|z|^{-3 / 2}+|\tilde{z}|^{-3 / 2}\right) .
\end{aligned}
$$

By symmetry, we deduce that

$$
\begin{aligned}
|\sigma(z)-\sigma(\tilde{z})| & \leq|z-\tilde{z}|\left(\frac{3}{2} \min (|z|,|\tilde{z}|) \max \left(|z|^{-5 / 2},|\tilde{z}|^{-5 / 2}\right)+|z|^{-3 / 2}+|\tilde{z}|^{-3 / 2}\right) \\
& \leq \frac{5}{2}|z-\tilde{z}|\left(|z|^{-3 / 2}+|\tilde{z}|^{-3 / 2}\right) .
\end{aligned}
$$

We also have $|b(z)| \leq 2|z|^{-2}$, so that $|b(z)-b(\tilde{z})| \leq 2\left(|z|^{-2}+|\tilde{z}|^{-2}\right)$. Finally,

$$
\begin{aligned}
|b(z)-b(\tilde{z})| & \leq\left. 2|| z\right|^{-3}-|\tilde{z}|^{-3}|\cdot| z|+2| z-\left.\tilde{z}|\cdot| \tilde{z}\right|^{-3} \\
& \leq 6|z| \max \left(|z|^{-4},|\tilde{z}|^{-4}\right)|z-\tilde{z}|+2|z-\tilde{z}|\left(|z|^{-3}+|\tilde{z}|^{-3}\right),
\end{aligned}
$$

whence by symmetry,

$$
\begin{aligned}
|b(z)-b(\tilde{z})| & \leq 6 \min (|z|,|\tilde{z}|) \max \left(|z|^{-4},|\tilde{z}|^{-4}\right)|z-\tilde{z}|+2|z-\tilde{z}|\left(|z|^{-3}+|\tilde{z}|^{-3}\right) \\
& \leq 8|z-\tilde{z}|\left(|z|^{-3}+|\tilde{z}|^{-3}\right)
\end{aligned}
$$

which ends the proof.

Next, we state some easy estimates of constant use in the paper. 
Lemma 4. Let $\alpha \in(-3,0]$. There is a constant $C_{\alpha}$ such that for all $g \in \mathcal{P} \cap L^{\infty}$, all $\epsilon \in(0,1]$,

$$
\begin{aligned}
& \sup _{v \in \mathbb{R}^{3}} \int_{\mathbb{R}^{3}}\left|v-v^{*}\right|^{\alpha} g\left(v^{*}\right) d v^{*} \leq 1+C_{\alpha}|| g \|_{\infty}, \\
& \int_{\mathbb{R}^{3}} \int_{\mathbb{R}^{3}}\left|v-v^{*}\right|^{\alpha} g(v) g\left(v^{*}\right) d v d v^{*} \leq 1+C_{\alpha}\|g\|_{\infty}, \\
& \sup _{v, w \in \mathbb{R}^{3}} \int_{\left|v-v^{*}\right| \leq \epsilon}\left|w-v^{*}\right|^{\alpha} g\left(v^{*}\right) d v^{*} \leq C_{\alpha}\|g\|_{\infty} \epsilon^{3+\alpha} .
\end{aligned}
$$

There is a constant $C$ such that for all $g \in \mathcal{P} \cap L^{\infty}$, all $\epsilon \in(0,1]$,

$$
\sup _{v \in \mathbb{R}^{3}} \int_{\left|v-v^{*}\right| \geq \epsilon}\left|v-v^{*}\right|^{-3} g\left(v^{*}\right) d v^{*} \leq 1+C|| g \|_{\infty} \log (1 / \epsilon) .
$$

Proof. Below, the variable $u$ belongs to $\mathbb{R}^{3}$. Recall that there is a constant $C$ such that for $\epsilon \in(0,1]$,

$$
\int_{\epsilon \leq|u| \leq 1}|u|^{-3} d u=C \log (1 / \epsilon)
$$

and that for $\alpha \in(-3,0]$, there is a constant $C_{\alpha}$ such that for all $\epsilon \in(0,1]$, all $u_{0} \in \mathbb{R}^{3}$,

$$
\int_{|u| \leq \epsilon}\left|u+u_{0}\right|^{\alpha} d u \leq C_{\alpha} \epsilon^{3+\alpha} .
$$

Since $g$ has mass 1 and $\alpha \in(-3,0]$, for any $v \in \mathbb{R}^{3}$,

$$
\begin{aligned}
\int_{\mathbb{R}^{3}}\left|v-v^{*}\right|^{\alpha} g\left(v^{*}\right) d v^{*} & \leq \int_{\left|v-v^{*}\right| \geq 1} g\left(v^{*}\right) d v^{*}+\int_{\left|v-v^{*}\right| \leq 1}\left|v-v^{*}\right|^{\alpha} g\left(v^{*}\right) d v^{*} \\
& \leq 1+\|g\|_{\infty} \int_{\left|v-v^{*}\right| \leq 1}\left|v-v^{*}\right|^{\alpha} d v^{*} \\
& =1+\|g\|_{\infty} \int_{|u| \leq 1}|u|^{\alpha} d u,
\end{aligned}
$$

whence (7) due to (12) with $\epsilon=1$. Inequality (8) follows from (7) because $g$ has mass 1 . Next (9) is deduced from (12): for $v, w \in \mathbb{R}^{3}$ and $\epsilon \in(0,1]$,

$$
\begin{aligned}
\int_{\left|v-v^{*}\right| \leq \epsilon}\left|w-v^{*}\right|^{\alpha} g\left(v^{*}\right) d v^{*} & \leq|| g \|_{\infty} \int_{\left|v-v^{*}\right| \leq \epsilon}\left|w-v^{*}\right|^{\alpha} d v^{*} \\
& =\|g\|_{\infty} \int_{|u| \leq \epsilon}|u+(v-w)|^{\alpha} d u .
\end{aligned}
$$

Finally, for any $v \in \mathbb{R}^{3}$,

$$
\begin{aligned}
\int_{\left|v-v^{*}\right| \geq \epsilon}\left|v-v^{*}\right|^{-3} g\left(v^{*}\right) d v^{*} & \leq \int_{\left|v-v^{*}\right| \geq 1} g\left(v^{*}\right) d v^{*}+\|g\|_{\infty} \int_{\epsilon \leq\left|v-v^{*}\right| \leq 1}\left|v-v^{*}\right|^{-3} d v^{*} \\
& \leq 1+\|g\|_{\infty} \int_{\epsilon \leq|u| \leq 1}|u|^{-3} d u
\end{aligned}
$$

from which (10) follows using (11).

We also consider the increasing continuous function $\Psi:[0, \infty) \mapsto \mathbb{R}_{+}$defined by

$$
\Psi(x)=x\left(1-\mathbf{1}_{\{x \leq 1\}} \log x\right) .
$$

The following remark will allow us to apply the Jensen inequality. 
Remark 5. We can find a concave increasing continuous function $\Gamma: \mathbb{R}_{+} \mapsto \mathbb{R}_{+}$such that for all $x \geq 0, \Psi(x) / 2 \leq \Gamma(x) \leq 2 \Psi(x)$.

Proof. Choose $\Gamma(x)=x(1-\log x)$ for $x \in[0,1 / 2]$ and $\Gamma(x)=x \log 2+1 / 2$ for $x \geq 1 / 2$.

The two next lemmas contain the fundamental computations of the paper.

Lemma 6. For any $g \in \mathcal{P} \cap L^{\infty}$, for all $v, \tilde{v} \in \mathbb{R}^{3}$,

$$
\begin{gathered}
\int_{\mathbb{R}^{3}}\left|\sigma\left(v-v^{*}\right)-\sigma\left(\tilde{v}-v^{*}\right)\right|^{2} g\left(v^{*}\right) d v^{*} \leq C\left(1+\|g\|_{\infty}\right) \Psi\left(|v-\tilde{v}|^{2}\right), \\
\int_{\mathbb{R}^{3}}\left|b\left(v-v^{*}\right)-b\left(\tilde{v}-v^{*}\right)\right| g\left(v^{*}\right) d v^{*} \leq C\left(1+\|g\|_{\infty}\right) \Psi(|v-\tilde{v}|) .
\end{gathered}
$$

Proof. We denote by $I$ the left hand side of (14). Using Lemma 3 ,

$$
\left|\sigma\left(v-v^{*}\right)-\sigma\left(\tilde{v}-v^{*}\right)\right|^{2} \leq C \min \left\{|v-\tilde{v}|^{2}\left(\left|v-v^{*}\right|^{-3}+\left|\tilde{v}-v^{*}\right|^{-3}\right) ;\left|v-v^{*}\right|^{-1}+\left|\tilde{v}-v^{*}\right|^{-1}\right\} .
$$

Thus

$$
\begin{aligned}
I \leq & C \mathbf{1}_{\{|v-\tilde{v}| \geq 1\}} \int_{\mathbb{R}^{3}}\left(\left|v-v^{*}\right|^{-1}+\left|\tilde{v}-v^{*}\right|^{-1}\right) g\left(v^{*}\right) d v^{*} \\
& +C \mathbf{1}_{\{|v-\tilde{v}| \leq 1\}} \int_{\mathbb{R}^{3}} \mathbf{1}_{\left\{\left|v-v^{*}\right| \geq|v-\tilde{v}|^{2},\left|\tilde{v}-v^{*}\right| \geq|v-\tilde{v}|^{2}\right\}}|v-\tilde{v}|^{2}\left(\left|v-v^{*}\right|^{-3}+\left|\tilde{v}-v^{*}\right|^{-3}\right) g\left(v^{*}\right) d v^{*} \\
& +C \mathbf{1}_{\{|v-\tilde{v}| \leq 1\}} \int_{\mathbb{R}^{3}} \mathbf{1}_{\left\{\left|v-v^{*}\right| \leq|v-\tilde{v}|^{2}\right\}}\left(\left|v-v^{*}\right|^{-1}+\left|\tilde{v}-v^{*}\right|^{-1}\right) g\left(v^{*}\right) d v^{*} \\
& +C \mathbf{1}_{\{|v-\tilde{v}| \leq 1\}} \int_{\mathbb{R}^{3}} \mathbf{1}_{\left\{\left|\tilde{v}-v^{*}\right| \leq|v-\tilde{v}|^{2}\right\}}\left(\left|v-v^{*}\right|^{-1}+\left|\tilde{v}-v^{*}\right|^{-1}\right) g\left(v^{*}\right) d v^{*} \\
= & : C\left(I_{1}+I_{2}+I_{3}+I_{4}\right) .
\end{aligned}
$$

First, (7) with $\alpha=-1$ implies that

$$
I_{1} \leq C\left(1+\|g\|_{\infty}\right) \mathbf{1}_{\{|v-\tilde{v}| \geq 1\}} \leq C\left(1+\|g\|_{\infty}\right) \Psi\left(|v-\tilde{v}|^{2}\right) .
$$

Next, using (10) with $\epsilon=|v-\tilde{v}|^{2}$ yields

$$
\begin{aligned}
I_{2} & \leq \mathbf{1}_{\{|v-\tilde{v}| \leq 1\}}|v-\tilde{v}|^{2}\left(\int_{\left|v-v^{*}\right| \geq|v-\tilde{v}|^{2}}\left|v-v^{*}\right|^{-3} g\left(v^{*}\right) d v^{*}+\int_{\left|\tilde{v}-v^{*}\right| \geq|v-\tilde{v}|^{2}}\left|\tilde{v}-v^{*}\right|^{-3} g\left(v^{*}\right) d v^{*}\right) \\
& \leq \mathbf{1}_{\{|v-\tilde{v}| \leq 1\}}|v-\tilde{v}|^{2}\left(2+C|| g \|_{\infty} \log \left(1 /|v-\tilde{v}|^{2}\right)\right) \\
& \leq C\left(1+\|g\|_{\infty}\right) \mathbf{1}_{\{|v-\tilde{v}| \leq 1\}}|v-\tilde{v}|^{2}\left(1-\log \left(|v-\tilde{v}|^{2}\right)\right) \\
& \leq C\left(1+\|g\|_{\infty}\right) \Psi\left(|v-\tilde{v}|^{2}\right) .
\end{aligned}
$$

Finally, we deduce from (9) with $\alpha=-1$ and $\epsilon=|v-\tilde{v}|^{2}$ that

$$
I_{3}+I_{4} \leq C|| g\left\|_{\infty} \mathbf{1}_{\{|v-\tilde{v}| \leq 1\}}\left(|v-\tilde{v}|^{2}\right)^{3-1} \leq C|| g\right\|_{\infty}|v-\tilde{v}|^{2} \leq C|| g \|_{\infty} \Psi\left(|v-\tilde{v}|^{2}\right) .
$$

We now denote by $J$ the left hand side of (15). By Lemma 3 ,

$$
\left|b\left(v-v^{*}\right)-b\left(\tilde{v}-v^{*}\right)\right| \leq C \min \left\{|v-\tilde{v}|\left(\left|v-v^{*}\right|^{-3}+\left|\tilde{v}-v^{*}\right|^{-3}\right) ;\left|v-v^{*}\right|^{-2}+\left|\tilde{v}-v^{*}\right|^{-2}\right\} .
$$


Thus

$$
\begin{aligned}
J \leq & C \mathbf{1}_{\{|v-\tilde{v}| \geq 1\}} \int_{\mathbb{R}^{3}}\left(\left|v-v^{*}\right|^{-2}+\left|\tilde{v}-v^{*}\right|^{-2}\right) g\left(v^{*}\right) d v^{*} \\
& +C \mathbf{1}_{\{|v-\tilde{v}| \leq 1\}} \int_{\mathbb{R}^{3}} \mathbf{1}_{\left\{\left|v-v^{*}\right| \geq|v-\tilde{v}|^{2},\left|\tilde{v}-v^{*}\right| \geq|v-\tilde{v}|^{2}\right\}}|v-\tilde{v}|\left(\left|v-v^{*}\right|^{-3}+\left|\tilde{v}-v^{*}\right|^{-3}\right) g\left(v^{*}\right) d v^{*} \\
& +C \mathbf{1}_{\{|v-\tilde{v}| \leq 1\}} \int_{\mathbb{R}^{3}} \mathbf{1}_{\left\{\left|v-v^{*}\right| \leq|v-\tilde{v}|^{2}\right\}}\left(\left|v-v^{*}\right|^{-2}+\left|\tilde{v}-v^{*}\right|^{-2}\right) g\left(v^{*}\right) d v^{*} \\
& +C \mathbf{1}_{\{|v-\tilde{v}| \leq 1\}} \int_{\mathbb{R}^{3}} \mathbf{1}_{\left\{\left|\tilde{v}-v^{*}\right| \leq|v-\tilde{v}|^{2}\right\}}\left(\left|v-v^{*}\right|^{-2}+\left|\tilde{v}-v^{*}\right|^{-2}\right) g\left(v^{*}\right) d v^{*} \\
=: & C\left(J_{1}+J_{2}+J_{3}+J_{4}\right) .
\end{aligned}
$$

Using (7) with $\alpha=-2$, we get

$$
J_{1} \leq C\left(1+\|g\|_{\infty}\right) \mathbf{1}_{\{|v-\tilde{v}| \geq 1\}} \leq C\left(1+\|g\|_{\infty}\right) \Psi(|v-\tilde{v}|) .
$$

Next, (10) with $\epsilon=|v-\tilde{v}|^{2}$ yields

$$
\begin{aligned}
J_{2} & \leq \mathbf{1}_{\{|v-\tilde{v}| \leq 1\}}\left(\int_{\left|v-v^{*}\right| \geq|v-\tilde{v}|^{2}}\left|v-v^{*}\right|^{-3} g\left(v^{*}\right) d v^{*}+\int_{\left|\tilde{v}-v^{*}\right| \geq|v-\tilde{v}|^{2}}\left|\tilde{v}-v^{*}\right|^{-3} g\left(v^{*}\right) d v^{*}\right) \\
& \leq \mathbf{1}_{\{|v-\tilde{v}| \leq 1\}|v-\tilde{v}|\left[2+C|| g \|_{\infty} \log \left(1 /|v-\tilde{v}|^{2}\right)\right]} \\
& \leq C\left(1+\|g\|_{\infty}\right) \mathbf{1}_{\{|v-\tilde{v}| \leq 1\}}|v-\tilde{v}|[1-\log (|v-\tilde{v}|)] \\
& \leq C\left(1+\|g\|_{\infty}\right) \Psi(|v-\tilde{v}|) .
\end{aligned}
$$

Finally, $J_{3}+J_{4} \leq C|| g\left\|_{\infty} \mathbf{1}_{\{|v-\tilde{v}| \leq 1\}}\left(|v-\tilde{v}|^{2}\right)^{3-2} \leq C|| g\right\|_{\infty} \Psi(|v-\tilde{v}|)$ by (9) with $\alpha=-2$ and $\epsilon=|v-\tilde{v}|^{2}$.

Lemma 7. Consider $g, \tilde{g} \in \mathcal{P}_{2} \cap L^{\infty}$ and $Q, R \in \mathcal{H}(g, \tilde{g})$. Then

$$
\begin{aligned}
& \int_{\mathbb{R}^{3} \times \mathbb{R}^{3}} \int_{\mathbb{R}^{3} \times \mathbb{R}^{3}}|v-\tilde{v}| \cdot\left|b\left(v-v^{*}\right)-b\left(\tilde{v}-\tilde{v}^{*}\right)\right| Q(d v, d \tilde{v}) R\left(d v^{*}, d \tilde{v}^{*}\right) \\
\leq & C\left(1+\|g+\tilde{g}\|_{\infty}\right)\left\{\Psi\left(\int_{\mathbb{R}^{3} \times \mathbb{R}^{3}}|v-\tilde{v}|^{2} Q(d v, d \tilde{v})\right)+\Psi\left(\int_{\mathbb{R}^{3} \times \mathbb{R}^{3}}\left|v^{*}-\tilde{v}^{*}\right|^{2} R\left(d v^{*}, d \tilde{v}^{*}\right)\right)\right\} .
\end{aligned}
$$

Proof. We denote by $K$ the left hand side of (16) and by $\delta\left(v, \tilde{v}, v^{*}, \tilde{v}^{*}\right)=|v-\tilde{v}| \cdot\left|b\left(v-v^{*}\right)-b\left(\tilde{v}-\tilde{v}^{*}\right)\right|$. Due to Lemma 3 , $\delta$ is smaller than

$$
C\left(|v-\tilde{v}|+\left|v^{*}-\tilde{v}^{*}\right|\right) \min \left\{\left(|v-\tilde{v}|+\left|v^{*}-\tilde{v}^{*}\right|\right)\left(\left|v-v^{*}\right|^{-3}+\left|\tilde{v}-\tilde{v}^{*}\right|^{-3}\right) ;\left|v-v^{*}\right|^{-2}+\left|\tilde{v}-\tilde{v}^{*}\right|^{-2}\right\} \text {. }
$$

Hence we can write

$$
\begin{aligned}
\delta\left(v, \tilde{v}, v^{*}, \tilde{v}^{*}\right) \leq & C \mathbf{1}_{\left\{|v-\tilde{v}|+\left|v^{*}-\tilde{v}^{*}\right| \geq 1\right\}}\left(|v-\tilde{v}|+\left|v^{*}-\tilde{v}^{*}\right|\right)\left(\left|v-v^{*}\right|^{-2}+\left|\tilde{v}-\tilde{v}^{*}\right|^{-2}\right) \\
& +C \mathbf{1}_{\left\{|v-\tilde{v}|+\left|v^{*}-\tilde{v}^{*}\right| \leq 1\right\}} \mathbf{1}_{\left\{\left|v-v^{*}\right| \geq|v-\tilde{v}|^{4},\left|\tilde{v}-\tilde{v}^{*}\right| \geq|v-\tilde{v}|^{4}\right\}}|v-\tilde{v}|^{2}\left(\left|v-v^{*}\right|^{-3}+\left|\tilde{v}-\tilde{v}^{*}\right|^{-3}\right) \\
& +C \mathbf{1}_{\left\{|v-\tilde{v}|+\left|v^{*}-\tilde{v}^{*}\right| \leq 1\right\}} \mathbf{1}_{\left\{\left|v-v^{*}\right| \geq|v-\tilde{v}|^{4},\left|\tilde{v}-\tilde{v}^{*}\right| \geq|v-\tilde{v}|^{4}\right\}}\left|v^{*}-\tilde{v}^{*}\right|^{2}\left(\left|v-v^{*}\right|^{-3}+\left|\tilde{v}-\tilde{v}^{*}\right|^{-3}\right) \\
& +C \mathbf{1}_{\left\{|v-\tilde{v}|+\left|v^{*}-\tilde{v}^{*}\right| \leq 1\right\}} \mathbf{1}_{\left\{\left|v-v^{*}\right| \leq|v-\tilde{v}|^{4}\right\}}\left(|v-\tilde{v}|+\left|v^{*}-\tilde{v}^{*}\right|\right)\left(\left|v-v^{*}\right|^{-2}+\left|\tilde{v}-\tilde{v}^{*}\right|^{-2}\right) \\
& +C \mathbf{1}_{\left\{|v-\tilde{v}|+\left|v^{*}-\tilde{v}^{*}\right| \leq 1\right\}} \mathbf{1}_{\left\{\left|\tilde{v}-\tilde{v}^{*}\right| \leq|v-\tilde{v}|^{4}\right\}}\left(|v-\tilde{v}|+\left|v^{*}-\tilde{v}^{*}\right|\right)\left(\left|v-v^{*}\right|^{-2}+\left|\tilde{v}-\tilde{v}^{*}\right|^{-2}\right) \\
& =: C \sum_{1}^{5} \delta_{i}\left(v, \tilde{v}, v^{*}, \tilde{v}^{*}\right) .
\end{aligned}
$$


Thus $K \leq C \sum_{1}^{5} K_{i}$, where $K_{i}=\int_{\mathbb{R}^{3} \times \mathbb{R}^{3}} \int_{\mathbb{R}^{3} \times \mathbb{R}^{3}} \delta_{i}\left(v, \tilde{v}, v^{*}, \tilde{v}^{*}\right) Q(d v, d \tilde{v}) R\left(d v^{*}, d \tilde{v}^{*}\right)$. First,

$$
\begin{aligned}
\delta_{1}\left(v, \tilde{v}, v^{*}, \tilde{v}^{*}\right) & \leq\left(|v-\tilde{v}|+\left|v^{*}-\tilde{v}^{*}\right|\right)^{2}\left(\left|v-v^{*}\right|^{-2}+\left|\tilde{v}-\tilde{v}^{*}\right|^{-2}\right) \\
& \leq 2\left(|v-\tilde{v}|^{2}+\left|v^{*}-\tilde{v}^{*}\right|^{2}\right)\left(\left|v-v^{*}\right|^{-2}+\left|\tilde{v}-\tilde{v}^{*}\right|^{-2}\right) .
\end{aligned}
$$

As a consequence,

$$
\begin{aligned}
K_{1} \leq & 2 \int_{\mathbb{R}^{3} \times \mathbb{R}^{3}}|v-\tilde{v}|^{2} Q(d v, d \tilde{v}) \int_{\mathbb{R}^{3} \times \mathbb{R}^{3}}\left(\left|v-v^{*}\right|^{-2}+\left|\tilde{v}-\tilde{v}^{*}\right|^{2}\right) R\left(d v^{*}, d \tilde{v}^{*}\right) \\
& +2 \int_{\mathbb{R}^{3} \times \mathbb{R}^{3}}\left|v^{*}-\tilde{v}^{*}\right|^{2} R\left(d v^{*}, d \tilde{v}^{*}\right) \int_{\mathbb{R}^{3} \times \mathbb{R}^{3}}\left(\left|v-v^{*}\right|^{-2}+\left|\tilde{v}-\tilde{v}^{*}\right|^{-2}\right) Q(d v, d \tilde{v}) . \\
= & 2 K_{1,1}+2 K_{1,2} .
\end{aligned}
$$

Since now $R$ has marginals $g$ and $\tilde{g}$, we deduce from (7) with $\alpha=-2$ that

$$
\begin{aligned}
K_{1,1} & =\int_{\mathbb{R}^{3} \times \mathbb{R}^{3}}|v-\tilde{v}|^{2} Q(d v, d \tilde{v})\left(\int_{\mathbb{R}^{3}}\left|v-v^{*}\right|^{-2} g\left(v^{*}\right) d v^{*}+\int_{\mathbb{R}^{3}}\left|\tilde{v}-\tilde{v}^{*}\right|^{2} \tilde{g}\left(\tilde{v}^{*}\right) d \tilde{v}^{*}\right) \\
& \leq \int_{\mathbb{R}^{3} \times \mathbb{R}^{3}}|v-\tilde{v}|^{2} Q(d v, d \tilde{v})\left[1+C|| g\left\|_{\infty}+1+C\right\| \tilde{g} \|_{\infty}\right] \\
& \leq C\left(1+\|g+\tilde{g}\|_{\infty}\right) \int_{\mathbb{R}^{3} \times \mathbb{R}^{3}}|v-\tilde{v}|^{2} Q(d v, d \tilde{v}) \\
& \leq C\left(1+\|g+\tilde{g}\|_{\infty}\right) \Psi\left(\int_{\mathbb{R}^{3} \times \mathbb{R}^{3}}|v-\tilde{v}|^{2} Q(d v, d \tilde{v})\right) .
\end{aligned}
$$

Similarly,

$$
K_{1,2} \leq C\left(1+\|g+\tilde{g}\|_{\infty}\right) \Psi\left(\int_{\mathbb{R}^{3} \times \mathbb{R}^{3}}\left|v^{*}-\tilde{v}^{*}\right|^{2} R\left(d v^{*}, d \tilde{v}^{*}\right)\right) .
$$

Next,

$$
\delta_{2}\left(v, \tilde{v}, v^{*}, \tilde{v}^{*}\right) \leq \mathbf{1}_{\{|v-\tilde{v}| \leq 1\}} \mathbf{1}_{\left\{\left|v-v^{*}\right| \geq|v-\tilde{v}|^{4},\left|\tilde{v}-\tilde{v}^{*}\right| \geq|v-\tilde{v}|^{4}\right\}}|v-\tilde{v}|^{2}\left(\left|v-v^{*}\right|^{-3}+\left|\tilde{v}-\tilde{v}^{*}\right|^{-3}\right) .
$$

Thus, (10) with $\epsilon=|v-\tilde{v}|^{4}$ yields

$$
\begin{aligned}
K_{2} & \leq \int_{\mathbb{R}^{3} \times \mathbb{R}^{3}} Q(d v, d \tilde{v}) \mathbf{1}_{\{|v-\tilde{v}| \leq 1\}}|v-\tilde{v}|^{2} \int_{\mathbb{R}^{3} \times \mathbb{R}^{3}} R\left(d v^{*}, d \tilde{v}^{*}\right) \\
& =\int_{\mathbb{R}^{3} \times \mathbb{R}^{3}} Q(d v, d \tilde{v}) \mathbf{1}_{\{|v-\tilde{v}| \leq 1\}}|v-\tilde{v}|^{2} \\
& \left(\int_{\left|v-v^{*}\right| \geq|v-\tilde{v}|^{4}}\left|v-v^{*}\right|^{-3} g\left(v^{*}\right) d v^{*}+\int_{\left|\tilde{v}-\tilde{v}^{*}\right| \geq|v-\tilde{v}|^{4}}\left|\tilde{v}-\tilde{v}^{*}\right|^{-3} \tilde{g}\left(\tilde{v}^{*}\right) d \tilde{v}^{*}\right) \\
& \leq \int_{\mathbb{R}^{3} \times \mathbb{R}^{3}} Q(d v, d \tilde{v}) \mathbf{1}_{\{|v-\tilde{v}| \leq 1\}}|v-\tilde{v}|^{2}\left[1+\left.C|| g\right|_{\infty} \log \left(1 /|v-\tilde{v}|^{4}\right)+1+C|| \tilde{g} \|_{\infty} \log \left(1 /|v-\tilde{v}|^{4}\right)\right] \\
& \left.\leq C\left(1+|| g+\tilde{g} \|_{\infty}\right) \int_{\mathbb{R}^{3} \times \mathbb{R}^{3}} Q(d v, d \tilde{v}) \mathbf{1}_{\left.\{|v-\tilde{v}| \leq 1\}|v-\tilde{v}|^{4}\right\}}\left|\tilde{v}-\tilde{v}^{*}\right|^{-3}\right) \\
& \leq C\left(1+\log \left(1 /|v-\tilde{v}|^{2}\right)\right] \\
&
\end{aligned}
$$


Remark 5 and the Jensen inequality allow us to conclude that

$$
K_{2} \leq C\left(1+\|g+\tilde{g}\|_{\infty}\right) \Psi\left(\int_{\mathbb{R}^{3} \times \mathbb{R}^{3}}|v-\tilde{v}|^{2} Q(d v, d \tilde{v})\right) .
$$

The third term $K_{3}$ is bounded symmetrically. For the fourth term, we first notice that

$$
\delta_{4}\left(v, \tilde{v}, v^{*}, \tilde{v}^{*}\right) \leq \mathbf{1}_{\{|v-\tilde{v}| \leq 1\}} \mathbf{1}_{\left\{\left|v-v^{*}\right| \leq|v-\tilde{v}|^{4}\right\}}\left[\left|v-v^{*}\right|^{-2}+\left|\tilde{v}-\tilde{v}^{*}\right|^{-2}\right],
$$

whence

$$
K_{4} \leq \int_{\mathbb{R}^{3} \times \mathbb{R}^{3}} Q(d v, d \tilde{v}) \mathbf{1}_{\{|v-\tilde{v}| \leq 1\}} \int_{\left|v-v^{*}\right| \leq|v-\tilde{v}|^{4}}\left[\left|v-v^{*}\right|^{-2}+\left|\tilde{v}-\tilde{v}^{*}\right|^{-2}\right] R\left(d v^{*}, d \tilde{v}^{*}\right) .
$$

But for all $v, \tilde{v}$, using (9) with $\alpha=-2$ and $\epsilon=|v-\tilde{v}|^{4}$,

$$
\int_{\left|v-v^{*}\right| \leq|v-\tilde{v}|^{4}}\left|v-v^{*}\right|^{-2} R\left(d v^{*}, d \tilde{v}^{*}\right)=\int_{\left|v-v^{*}\right| \leq|v-\tilde{v}|^{4}}\left|v-v^{*}\right|^{-2} g\left(v^{*}\right) d v^{*} \leq C|| g \|_{\infty}|v-\tilde{v}|^{4} .
$$

Using now the Hölder inequality (with $p=5$ and $q=5 / 4$ ), then (9) with $\alpha=0, \epsilon=|v-\tilde{v}|^{4}$ and finally (7) with $\alpha=-5 / 2$,

$$
\begin{aligned}
& \int_{\left|v-v^{*}\right| \leq|v-\tilde{v}|^{4}}\left|\tilde{v}-\tilde{v}^{*}\right|^{-2} R\left(d v^{*}, d \tilde{v}^{*}\right) \\
\leq & \left(\int_{\left|v-v^{*}\right| \leq|v-\tilde{v}|^{4}} R\left(d v^{*}, d \tilde{v}^{*}\right)\right)^{1 / 5}\left(\int_{\mathbb{R}^{3} \times \mathbb{R}^{3}}\left|\tilde{v}-\tilde{v}^{*}\right|^{-5 / 2} R\left(d v^{*}, d \tilde{v}^{*}\right)\right)^{4 / 5} \\
= & \left(\int_{\left|v-v^{*}\right| \leq|v-\tilde{v}|^{4}} g\left(v^{*}\right) d v^{*}\right)^{1 / 5}\left(\int_{\mathbb{R}^{3}}\left|\tilde{v}-\tilde{v}^{*}\right|^{-5 / 2} \tilde{g}\left(\tilde{v}^{*}\right) d \tilde{v}^{*}\right)^{4 / 5} \\
\leq & \left(C|| g \|_{\infty}\left[|v-\tilde{v}|^{4}\right]^{3}\right)^{1 / 5}\left(1+C \mid \tilde{g} \|_{\infty}\right)^{4 / 5} \\
\leq & C\left(1+\| g+\tilde{g}||_{\infty}\right)|v-\tilde{v}|^{12 / 5},
\end{aligned}
$$

because $\|g\|_{\infty}^{1 / 5}\left(1+\|\tilde{g}\|_{\infty}\right)^{4 / 5} \leq 1+\|g\|_{\infty}+\|\tilde{g}\|_{\infty} \leq 1+2\|g+\tilde{g}\|_{\infty}$. We thus have shown that

$$
\begin{aligned}
K_{4} & \leq C\left(1+\|g+\tilde{g}\|_{\infty}\right) \int_{\mathbb{R}^{3} \times \mathbb{R}^{3}} Q(d v, d \tilde{v}) \mathbf{1}_{\{|v-\tilde{v}| \leq 1\}}\left(|v-\tilde{v}|^{4}+|v-\tilde{v}|^{12 / 5}\right) \\
& \leq C\left(1+\|g+\tilde{g}\|_{\infty}\right) \int_{\mathbb{R}^{3} \times \mathbb{R}^{3}} Q(d v, d \tilde{v})|v-\tilde{v}|^{2} \\
& \leq C\left(1+\|g+\tilde{g}\|_{\infty}\right) \Psi\left(\int_{\mathbb{R}^{3} \times \mathbb{R}^{3}}|v-\tilde{v}|^{2} Q(d v, d \tilde{v})\right) .
\end{aligned}
$$

The last term $K_{5}$ is treated symmetrically, which ends the proof.

We conclude this section by recalling a generalization of the Gronwall Lemma of which the proof can be found in Chemin [4, Lemme 5.2.1 page 89].

Lemma 8. Let $T>0$ and $\gamma:[0, T] \mapsto \mathbb{R}_{+}$satisfy $\int_{0}^{T} \gamma(s) d s<\infty$. Recall that $\Psi$ was defined by (13) and set $M(x)=\int_{x}^{1}(1 / \Psi(y)) d y$ for $x>0$. Consider a bounded measurable function $\rho:[0, T] \mapsto \mathbb{R}_{+}$ such that, for some $a \geq 0$, for all $t \in[0, T], \rho(t) \leq a+\int_{0}^{t} \gamma(s) \Psi(\rho(s)) d s$.

(i) If $a=0$, then $\rho(t)=0$ for all $t \in[0, T]$.

(ii) If $a>0$, then $M(a)-M(\rho(t)) \leq \int_{0}^{t} \gamma(s) d s$ for all $t \in[0, T]$. 


\section{An INTEGRAL INEQUALity}

Theorem 2 will be easily deduced, in the next section, from Lemma 8 and the following result. Recall that $\Psi$ was defined in (13).

Theorem 9. There is a constant $C$ such that for any pair of weak solutions $\left(f_{t}\right)_{t \in[0, T]}$ and $\left(\tilde{f}_{t}\right)_{t \in[0, T]}$ to (1), there is a bounded function $\rho:[0, T] \mapsto \mathbb{R}_{+}$satisfying, for all $t \in[0, T]$,

$$
\mathcal{W}_{2}^{2}\left(f_{t}, \tilde{f}_{t}\right) \leq \rho(t) \quad \text { and } \quad \rho(t) \leq \mathcal{W}_{2}^{2}\left(f_{0}, \tilde{f}_{0}\right)+C \int_{0}^{t}\left(1+\left\|f_{s}+\tilde{f}_{s}\right\|_{\infty}\right) \Psi(\rho(s)) d s
$$

From now on, $T>0$ and the two weak solutions $\left(f_{t}\right)_{t \in[0, T]},\left(\tilde{f}_{t}\right)_{t \in[0, T]}$ to (1), both belonging to $L^{\infty}\left([0, T], \mathcal{P}_{2}\right) \cap L^{1}\left([0, T], L^{\infty}\right)$ are fixed. We follow closely the scheme of proof of [8]: first, we introduce two coupled Landau stochastic processes, the first one associated with $f$, the second one associated with $\tilde{f}$, in such a way that they remain as close to each other as possible. The probabilistic interpretation of the Landau equation we use here has been introduced by Funaki [9], Guérin 10 and is inspired by the work of Tanaka [12.

For all $s \in[0, T]$, we denote by $R_{s} \in \mathcal{H}\left(f_{s}, \tilde{f}_{s}\right)$ the (unique) probability measure on $\mathbb{R}^{3} \times \mathbb{R}^{3}$ with marginals $f_{s}$ and $\tilde{f}_{s}$ such that $\mathcal{W}_{2}^{2}\left(f_{s}, \tilde{f}_{s}\right)=\int_{\mathbb{R}^{3} \times \mathbb{R}^{3}}|v-\tilde{v}|^{2} R_{s}(d v, d \tilde{v})$.

On some probability space, we consider a three-dimensional white noise $W(d v, d \tilde{v}, d s)$ on $\mathbb{R}^{3} \times$ $\mathbb{R}^{3} \times[0, T]$ with covariance measure $R_{s}(d v, d \tilde{v}) d s$. This means that $W=\left(W_{1}, W_{2}, W_{3}\right)$, where $W_{1}, W_{2}$ and $W_{3}$ are three independent white noises on $\mathbb{R}^{3} \times \mathbb{R}^{3} \times[0, T]$ with covariance measure $R_{s}(d v, d \tilde{v}) d s$, see Walsh [17] for definitions. We also need two $\mathbb{R}^{3}$-valued random variables $V_{0}, \tilde{V}_{0}$ with laws $f_{0}, \tilde{f}_{0}$, independent of $W$, such that $\mathcal{W}_{2}^{2}\left(f_{0}, \tilde{f}_{0}\right)=\mathbb{E}\left[\left|V_{0}-\tilde{V}_{0}\right|^{2}\right]$. We consider the two following $\mathbb{R}^{3}$-valued stochastic differential equations.

$$
\begin{aligned}
& V_{t}=V_{0}+\int_{0}^{t} \int_{\mathbb{R}^{3} \times \mathbb{R}^{3}} \sigma\left(V_{s}-v\right) W(d v, d \tilde{v}, d s)+\int_{0}^{t} \int_{\mathbb{R}^{3}} b\left(V_{s}-v\right) f_{s}(v) d v d s \\
& \tilde{V}_{t}=\tilde{V}_{0}+\int_{0}^{t} \int_{\mathbb{R}^{3} \times \mathbb{R}^{3}} \sigma\left(\tilde{V}_{s}-\tilde{v}\right) W(d v, d \tilde{v}, d s)+\int_{0}^{t} \int_{\mathbb{R}^{3}} b\left(\tilde{V}_{s}-\tilde{v}\right) \tilde{f}_{s}(\tilde{v}) d \tilde{v} d s
\end{aligned}
$$

$b, \sigma$ being defined by (4) and (6) . We set $\mathcal{F}_{t}=\sigma\left\{V_{0}, \tilde{V}_{0}, W([0, s] \times A), s \in[0, t], A \in \mathcal{B}\left(\mathbb{R}^{3} \times \mathbb{R}^{3}\right)\right\}$.

Proposition 10. (i) There exists a unique pair $\left(V_{t}\right)_{t \in[0, T]}$, $\left(\tilde{V}_{t}\right)_{t \in[0, T]}$ of continuous $\left(\mathcal{F}_{t}\right)_{t \in[0, T]^{-}}$ adapted processes solving (17) and (18).

(ii) For all $t \in[0, T], \mathcal{L}\left(V_{t}\right)=f_{t}$ and $\mathcal{L}\left(\tilde{V}_{t}\right)=\tilde{f}_{t}$.

We admit this proposition for a while.

Proof of Theorem 9. By Proposition [10.(ii), we have $\mathcal{W}_{2}^{2}\left(f_{t}, \tilde{f}_{t}\right) \leq \mathbb{E}\left[\left|V_{t}-\tilde{V}_{t}\right|^{2}\right]$. We thus compute this last quantity carefully. The marginals of $R_{s}$ being $f_{s}$ and $\tilde{f}_{s}$, we may rewrite (17) and (18) as

$$
\begin{aligned}
& V_{t}=V_{0}+\int_{0}^{t} \int_{\mathbb{R}^{3} \times \mathbb{R}^{3}} \sigma\left(V_{s}-v\right) W(d v, d \tilde{v}, d s)+\int_{0}^{t} \int_{\mathbb{R}^{3} \times \mathbb{R}^{3}} b\left(V_{s}-v\right) R_{s}(d v, d \tilde{v}) d s \\
& \tilde{V}_{t}=\tilde{V}_{0}+\int_{0}^{t} \int_{\mathbb{R}^{3} \times \mathbb{R}^{3}} \sigma\left(\tilde{V}_{s}-\tilde{v}\right) W(d v, d \tilde{v}, d s)+\int_{0}^{t} \int_{\mathbb{R}^{3} \times \mathbb{R}^{3}} b\left(\tilde{V}_{s}-\tilde{v}\right) R_{s}(d v, d \tilde{v}) d s .
\end{aligned}
$$


Using the Itô formula and taking expectations, we obtain

$$
\begin{aligned}
\mathbb{E}\left[\left|V_{t}-\tilde{V}_{t}\right|^{2}\right]= & \mathbb{E}\left[\left|V_{0}-\tilde{V}_{0}\right|^{2}\right]+\sum_{i, l=1}^{3} \int_{0}^{t} \int_{\mathbb{R}^{3} \times \mathbb{R}^{3}} \mathbb{E}\left[\left(\sigma_{i l}\left(V_{s}-v\right)-\sigma_{i l}\left(\tilde{V}_{s}-\tilde{v}\right)\right)^{2}\right] R_{s}(d v, d \tilde{v}) d s \\
& +2 \int_{0}^{t} \int_{\mathbb{R}^{3} \times \mathbb{R}^{3}} \mathbb{E}\left[\left(b\left(V_{s}-v\right)-b\left(\tilde{V}_{s}-\tilde{v}\right)\right) \cdot\left(V_{s}-\tilde{V}_{s}\right)\right] R_{s}(d v, d \tilde{v}) d s \\
= & \mathcal{W}_{2}^{2}\left(f_{0}, \tilde{f}_{0}\right)+\int_{0}^{t} A_{s} d s+2 \int_{0}^{t} B_{s} d s .
\end{aligned}
$$

Let $Q_{s}(d v, d \tilde{v})$ be the law of the couple $\left(V_{s}, \tilde{V}_{s}\right)$. Using (16) and that $R_{s}, Q_{s} \in \mathcal{H}\left(f_{s}, \tilde{f}_{s}\right)$,

$$
\begin{aligned}
B_{s} & =\int_{\mathbb{R}^{3} \times \mathbb{R}^{3}} \int_{\mathbb{R}^{3} \times \mathbb{R}^{3}}|v-\tilde{v}| \cdot\left|b\left(v-v^{*}\right)-b\left(\tilde{v}-\tilde{v}^{*}\right)\right| Q_{s}(d v, d \tilde{v}) R_{s}\left(d v^{*}, d \tilde{v}^{*}\right) \\
& \leq C\left(1+\left\|f_{s}+\tilde{f}_{s}\right\|_{\infty}\right)\left\{\Psi\left(\int_{\mathbb{R}^{3} \times \mathbb{R}^{3}}|v-\tilde{v}|^{2} Q_{s}(d v, d \tilde{v})\right)+\Psi\left(\int_{\mathbb{R}^{3} \times \mathbb{R}^{3}}\left|v^{*}-\tilde{v}^{*}\right|^{2} R_{s}\left(d v^{*}, d \tilde{v}^{*}\right)\right)\right\} .
\end{aligned}
$$

Next, using (14),

$$
\begin{aligned}
A_{s}= & \int_{\mathbb{R}^{3} \times \mathbb{R}^{3}} \int_{\mathbb{R}^{3} \times \mathbb{R}^{3}}\left|\sigma\left(v-v^{*}\right)-\sigma\left(\tilde{v}-\tilde{v}^{*}\right)\right|^{2} Q_{s}(d v, d \tilde{v}) R_{s}\left(d v^{*}, d \tilde{v}^{*}\right) \\
\leq & 2 \int_{\mathbb{R}^{3} \times \mathbb{R}^{3}} \int_{\mathbb{R}^{3} \times \mathbb{R}^{3}}\left|\sigma\left(v-v^{*}\right)-\sigma\left(v-\tilde{v}^{*}\right)\right|^{2} Q_{s}(d v, d \tilde{v}) R_{s}\left(d v^{*}, d \tilde{v}^{*}\right) \\
& +2 \int_{\mathbb{R}^{3} \times \mathbb{R}^{3}} \int_{\mathbb{R}^{3} \times \mathbb{R}^{3}}\left|\sigma\left(v-\tilde{v}^{*}\right)-\sigma\left(\tilde{v}-\tilde{v}^{*}\right)\right|^{2} Q_{s}(d v, d \tilde{v}) R_{s}\left(d v^{*}, d \tilde{v}^{*}\right) \\
= & 2 \int_{\mathbb{R}^{3} \times \mathbb{R}^{3}} \int_{\mathbb{R}^{3}}\left|\sigma\left(v-v^{*}\right)-\sigma\left(v-\tilde{v}^{*}\right)\right|^{2} f_{s}(v) d v R_{s}\left(d v^{*}, d \tilde{v}^{*}\right) \\
& +2 \int_{\mathbb{R}^{3} \times \mathbb{R}^{3}} \int_{\mathbb{R}^{3}}\left|\sigma\left(v-\tilde{v}^{*}\right)-\sigma\left(\tilde{v}-\tilde{v}^{*}\right)\right|^{2} \tilde{f}_{s}\left(d \tilde{v}^{*}\right) Q_{s}(d v, d \tilde{v}) \\
\leq & C\left(1+\left\|f_{s}\right\|_{\infty}\right) \int_{\mathbb{R}^{3} \times \mathbb{R}^{3}} \Psi\left(\left|v^{*}-\tilde{v}^{*}\right|^{2}\right) R_{s}\left(d v^{*}, d \tilde{v}^{*}\right) \\
& +C\left(1+\left\|\tilde{f}_{s}\right\|_{\infty}\right) \int_{\mathbb{R}^{3} \times \mathbb{R}^{3}} \Psi\left(|v-\tilde{v}|^{2}\right) Q_{s}(d v, d \tilde{v}) .
\end{aligned}
$$

Due to Remark 5 and the Jensen inequality,

$$
A_{s} \leq C\left(1+\left\|f_{s}+\tilde{f}_{s}\right\|_{\infty}\right)\left\{\Psi\left(\int_{\mathbb{R}^{3} \times \mathbb{R}^{3}}|v-\tilde{v}|^{2} Q_{s}(d v, d \tilde{v})\right)+\Psi\left(\int_{\mathbb{R}^{3} \times \mathbb{R}^{3}}\left|v^{*}-\tilde{v}^{*}\right|^{2} R_{s}\left(d v^{*}, d \tilde{v}^{*}\right)\right)\right\}
$$

We now set $\rho(t):=\mathbb{E}\left[\left|V_{s}-\tilde{V}_{s}\right|^{2}\right]$. Since

$$
\mathcal{W}_{2}^{2}\left(f_{s}, \tilde{f}_{s}\right)=\int_{\mathbb{R}^{3} \times \mathbb{R}^{3}}\left|v^{*}-\tilde{v}^{*}\right|^{2} R_{s}\left(d v^{*}, d \tilde{v}^{*}\right) \leq \int_{\mathbb{R}^{3} \times \mathbb{R}^{3}}|v-\tilde{v}|^{2} Q_{s}(d v, d \tilde{v})=\rho(t),
$$

and since $\Psi$ is increasing, we have shown that

$$
\mathcal{W}_{2}^{2}\left(f_{t}, \tilde{f}_{t}\right) \leq \rho(t) \leq \mathcal{W}_{2}^{2}\left(f_{0}, \tilde{f}_{0}\right)+C \int_{0}^{t}\left(1+\left\|f_{s}+\tilde{f}_{s}\right\|_{\infty}\right) \Psi(\rho(s)) d s .
$$

It only remains to check that $\rho$ is bounded on $[0, T]$. But $\rho(t) \leq 2 \mathbb{E}\left[\left|V_{t}\right|^{2}\right]+2 \mathbb{E}\left[\left|\tilde{V}_{t}\right|^{2}\right]=2 m_{2}\left(f_{t}\right)+$ $2 m_{2}\left(\tilde{f}_{t}\right)$ by Proposition 10\}(ii) and $\left(f_{t}\right)_{t \in[0, T]},\left(\tilde{f}_{t}\right)_{t \in[0, T]} \in L^{\infty}\left([0, T], \mathcal{P}_{2}\right)$ by assumption. 
It remains to give the

Proof of Proposition 10. We only check the results for (17), the study of (18) being the same.

Step 1. For $x_{0} \in \mathbb{R}^{3}$ and for $X=\left(X_{t}\right)_{t \in[0, T]}$ a $\mathbb{R}^{3}$-valued progressively measurable process, we introduce the $\mathbb{R}^{3}$-valued progressively measurable process $\left(\Phi\left(x_{0}, X\right)_{t}\right)_{t \in[0, T]}$ defined by

$$
\Phi\left(x_{0}, X\right)_{t}=x_{0}+\int_{0}^{t} \int_{\mathbb{R}^{3} \times \mathbb{R}^{3}} \sigma\left(X_{s}-v\right) W(d v, d \tilde{v}, d s)+\int_{0}^{t} \int_{\mathbb{R}^{3}} b\left(X_{s}-v\right) f_{s}(v) d v d s .
$$

The goal of this step is to prove that $\left(\Phi\left(x_{0}, X\right)_{t}\right)_{t \in[0, T]}$ is automatically continuous and that

$$
\mathbb{E}\left[\sup _{[0, T]}\left|\Phi\left(x_{0}, X\right)_{t}\right|^{2}\right] \leq C\left(\left|x_{0}\right|^{2}+\int_{0}^{T}\left\|f_{s}\right\|_{\infty} d s+\left(\int_{0}^{T}\left\|f_{s}\right\|_{\infty} d s\right)^{2}\right),
$$

which is finite thanks to the conditions imposed $f$. We observe, since the first marginal of $R_{s}$ is $f_{s}$ and using (7) with $\alpha=-1$, that a.s.,

$$
\int_{0}^{T} \int_{\mathbb{R}^{3} \times \mathbb{R}^{3}}\left|\sigma\left(X_{s}-v\right)\right|^{2} R_{s}(d v, d \tilde{v}) d s \leq \int_{0}^{T} \int_{\mathbb{R}^{3}}\left|X_{s}-v\right|^{-1} f_{s}(v) d v d s \leq \int_{0}^{T} C\left(1+\left\|f_{s}\right\|_{\infty}\right) d s<\infty .
$$

From (7) with $\alpha=-2$,

$$
\int_{0}^{T} \int_{\mathbb{R}^{3}}\left|b\left(X_{s}-v\right)\right| f_{s}(v) d v d s \leq \int_{0}^{T} \int_{\mathbb{R}^{3}}\left|X_{s}-v\right|^{-2} f_{s}(v) d v d s \leq \int_{0}^{T} C\left(1+\left\|f_{s}\right\|_{\infty}\right) d s<\infty .
$$

The a.s. continuity of $\Phi\left(x_{0}, X\right)$ on $[0, T]$ follows and the mean square estimate is easily deduced from the Doob inequality.

Step 2. We now aim to show that for $t \in[0, T]$, for $X, Y$ two progressively measurable processes,

$$
\begin{aligned}
\Delta_{t} & :=\mathbb{E}\left[\left|\Phi\left(x_{0}, X\right)_{t}-\Phi\left(x_{0}, Y\right)_{t}\right|^{2}\right] \\
& \leq C \int_{0}^{t}\left(1+|| f_{s} \|_{\infty}\right)\left\{\Psi\left(\mathbb{E}\left[\left|\Phi\left(x_{0}, X\right)_{s}-\Phi\left(x_{0}, Y\right)_{s}\right|^{2}\right]\right)+\Psi\left(\mathbb{E}\left[\left|X_{s}-Y_{s}\right|^{2}\right]\right)\right\} d s,
\end{aligned}
$$

where $\Psi$ was defined in (13). Using the Itô formula and taking expectations, we derive

$$
\begin{aligned}
\Delta_{t}= & \sum_{i, l=1}^{3} \int_{0}^{t} \int_{\mathbb{R}^{3} \times \mathbb{R}^{3}} \mathbb{E}\left[\left(\sigma_{i l}\left(X_{s}-v\right)-\sigma_{i l}\left(Y_{s}-v\right)\right)^{2}\right] R_{s}(d v, d \tilde{v}) d s \\
& +2 \int_{0}^{t} \int_{\mathbb{R}^{3} \times \mathbb{R}^{3}} \mathbb{E}\left[\left(b\left(X_{s}-v\right)-b\left(Y_{s}-v\right)\right) \cdot\left(\Phi\left(x_{0}, X\right)_{s}-\Phi\left(x_{0}, Y\right)_{s}\right)\right] f_{s}(v) d v d s .
\end{aligned}
$$

Due to (14) and (15) and since the first marginal of $R_{s}$ is $f_{s}$,

$$
\begin{aligned}
\Delta_{t} \leq & C \int_{0}^{t} \mathbb{E}\left[\int_{\mathbb{R}^{3}}\left|\sigma\left(X_{s}-v\right)-\sigma\left(Y_{s}-v\right)\right|^{2} f_{s}(v) d v\right] d s \\
& +C \int_{0}^{t} \mathbb{E}\left[\left|\Phi\left(x_{0}, X\right)_{s}-\Phi\left(x_{0}, Y\right)_{s}\right| \int_{\mathbb{R}^{3}}\left|b\left(X_{s}-v\right)-b\left(Y_{s}-v\right)\right| f_{s}(v) d v\right] d s \\
\leq & C \int_{0}^{t}\left(1+|| f_{s} \|_{\infty}\right) \mathbb{E}\left[\Psi\left(\left|X_{s}-Y_{s}\right|^{2}\right)+\left|\Phi\left(x_{0}, X\right)_{s}-\Phi\left(x_{0}, Y\right)_{s}\right| \Psi\left(\left|X_{s}-Y_{s}\right|\right)\right] d s .
\end{aligned}
$$


But since $x \mapsto \Psi(x)$ is non-decreasing and $x \Psi(x) \leq \Psi\left(x^{2}\right)$, one has, for all $u, v \geq 0$,

$$
u \Psi(v) \leq \mathbf{1}_{\{u \leq v\}} v \Psi(v)+\mathbf{1}_{\{u \geq v\}} u \Psi(u) \leq \Psi\left(u^{2}\right)+\Psi\left(v^{2}\right) .
$$

We thus obtain

$$
\begin{aligned}
\Delta_{t} & \leq C \int_{0}^{t}\left(1+\left\|f_{s}\right\|_{\infty}\right) \mathbb{E}\left[\Psi\left(\left|X_{s}-Y_{s}\right|^{2}\right)+\Psi\left(\left|\Phi\left(x_{0}, X\right)_{s}-\Phi\left(x_{0}, Y\right)_{s}\right|^{2}\right)\right] d s \\
& \leq C \int_{0}^{t}\left(1+\left\|f_{s}\right\|_{\infty}\right)\left\{\Psi\left(\mathbb{E}\left[\left|X_{s}-Y_{s}\right|^{2}\right]\right)+\Psi\left(\mathbb{E}\left[\left|\Phi\left(x_{0}, X\right)_{s}-\Phi\left(x_{0}, Y\right)_{s}\right|^{2}\right]\right)\right\} d s
\end{aligned}
$$

the last inequality following from Remark 5 and the Jensen inequality.

Step 3. We now check the uniqueness for (17). Consider two solutions $V=\Phi\left(V_{0}, V\right)$ and $\tilde{V}=\Phi\left(V_{0}, \tilde{V}\right)$, and set $\rho(t)=\mathbb{E}\left[\left|V_{t}-\tilde{V}_{t}\right|^{2}\right]$, which is bounded on $[0, T]$ due to Step 1 . Using Step 2 , we deduce that

$$
\rho(t) \leq \int_{0}^{t} \gamma(s) \Psi(\rho(s)) d s
$$

where $\gamma(s)=C\left(1+\left\|f_{s}\right\|_{\infty}\right) \in L^{1}([0, T])$. Lemma 8 yields that $\rho(t)=0$, whence $V_{t}=\tilde{V}_{t}$ a.s., for all $t \in[0, T]$. The continuity obtained in Step 1 guarantees us that a.s., $\left(V_{t}\right)_{t \in[0, T]}=\left(\tilde{V}_{t}\right)_{t \in[0, T]}$.

Step 4. We now prove the existence of a solution to (17) using a Picard iteration. We define $V^{0}$ by $V_{t}^{0}=V_{0}$ and then by induction $V^{n+1}=\Phi\left(V_{0}, V^{n}\right)$. We then set $\rho_{n, k}(t)=\sup _{[0, t]} \mathbb{E}\left[\left|V_{s}^{n+k}-V_{s}^{n}\right|^{2}\right]$, which is uniformly bounded on $[0, T]$ due to Step 1 . Step 2 yields

$$
\rho_{n+1, k}(t) \leq \int_{0}^{t} \gamma(s)\left[\Psi\left(\rho_{n+1, k}(s)\right)+\Psi\left(\rho_{n, k}(s)\right)\right] d s,
$$

where $\gamma(s)=C\left(1+\left\|f_{s}\right\|_{\infty}\right) \in L^{1}([0, T])$. Thus for $\rho_{n}(t):=\sup _{k} \rho_{n, k}(t)$, we get $\rho_{n+1}(t) \leq$ $\int_{0}^{t} \gamma(s)\left[\Psi\left(\rho_{n}(s)\right)+\Psi\left(\rho_{n+1}(s)\right)\right] d s$. Finally, setting $\rho(t):=\lim \sup _{n} \rho_{n}(t)$, we deduce that $\rho(t) \leq$ $2 \int_{0}^{t} \gamma(s) \Psi(\rho(s)) d s$, whence $\rho(T)=0$ by Lemma 8. We have proved that

$$
\limsup _{n} \sup _{k} \sup _{[0, T]} \mathbb{E}\left[\left|V_{t}^{n+k}-V_{t}^{n}\right|^{2}\right]=0,
$$

so that the sequence $\left(V_{t}^{n}\right)_{t \in[0, T]}$ is Cauchy in $L^{\infty}\left([0, T], L^{2}(\Omega)\right)$. Hence there is a process $\left(V_{t}\right)_{t \in[0, T]}$ such that $\lim _{n} \sup _{[0, T]} \mathbb{E}\left[\left|V_{t}-V_{t}^{n}\right|^{2}\right]=0$. To conclude this step, it suffices to prove that $\kappa_{n}(t):=$ $\mathbb{E}\left[\left|\Phi\left(V_{0}, V^{n}\right)_{t}-\Phi\left(V_{0}, V\right)_{t}\right|^{2}\right]$ tends to 0 for each $t \in[0, T]$. This will allow us to pass to the limit in $V^{n+1}=\Phi\left(V_{0}, V^{n}\right)$ an to get $V=\Phi\left(V_{0}, V\right)$, so that $V$ will solve (17). The a.s. continuity of $V$ will then be deduced from Step 1.

We set $\epsilon_{n}:=\sup _{[0, T]} \mathbb{E}\left[\Psi\left(\left|V_{s}^{n}-V_{s}\right|^{2}\right)\right]$, which tends to 0 by Remark 5 and the Jensen inequality. Using Step 2 again, we immediately obtain, for $t \in[0, T]$,

$$
\kappa_{n}(t) \leq \int_{0}^{t} \gamma(s)\left(\epsilon_{n}+\Psi\left(\kappa_{n}(s)\right)\right) d s .
$$

For $\kappa(t)=\limsup _{n} \kappa_{n}(t)$ (which is bounded due to Step 1), we get $\kappa(t) \leq \int_{0}^{t} \gamma(s) \Psi(\kappa(s)) d s$. Lemma 8 thus yields $\kappa(t)=0$ for all $t \in[0, T]$, which concludes this step.

Step 5. It remains to prove that for all $s \in[0, T], \mathcal{L}\left(V_{s}\right)=f_{s}$, for $V$ the unique solution of (17). We set $g_{s}=\mathcal{L}\left(V_{s}\right)$ for all $s \in[0, T]$ and we first observe that $\left(g_{t}\right)_{t \in[0, T]}$ solves the linear Landau 
equation: for any $\varphi \in C_{b}^{2}\left(\mathbb{R}^{3}\right)$,

$$
\int_{\mathbb{R}^{3}} \varphi(x) g_{t}(d x)=\int_{\mathbb{R}^{3}} \varphi(x) f_{0}(d x)+\int_{0}^{t} \iint_{\mathbb{R}^{3} \times \mathbb{R}^{3}} L \varphi(x, v) g_{s}(d x) f_{s}(v) d v d s .
$$

with $L$ defined by (3). It suffices to apply the Itô formula, to take expectations, to use that the first marginal of $R_{s}$ is $f_{s}$ and that $\sigma \cdot \sigma^{t}=a$. See [8, around Eq. (2.4)] for the detailed computation.

Assume for a moment that there is uniqueness for the linear equation 19] Since $\left(f_{t}\right)_{t \in[0, T]}$ is a weak solution to (1), is also a weak solution to (19). We deduce that for all $t \in[0, T], g_{t}=f_{t}$.

To prove the uniqueness for (19), we use a result of Horowitz-Karandikar [11, Theorem B.1], see also Bath-Karandikar [3, Theorem 5.2]. Consider, for $t \in[0, T], x \in \mathbb{R}^{3}$ and $\varphi \in C_{b}^{2}$, the operator $\mathcal{A}_{t} \varphi(x):=\int_{\mathbb{R}^{3}} L \varphi(x, v) f_{t}(v) d v$. A stochastic process $\left(X_{t}\right)_{t \in\left[t_{0}, T\right]}$ is said to solve the martingale problem for $\left(C_{b}^{2}, \mathcal{A}_{t}\right)$ if for all $\varphi \in C_{b}^{2}$, the process $\varphi\left(X_{t}\right)-\int_{t_{0}}^{t} \mathcal{A}_{s} \varphi\left(X_{s}\right) d s$ defined for $t \in\left[t_{0}, T\right]$ is a martingale. To apply [11, Theorem B.1], we have to check that:

(i) there is a countable family $\left(\varphi_{k}\right)_{k \geq 1} \subset C_{b}^{2}$ such that for all $t \in[0, T],\left\{\left(\varphi_{k}, \mathcal{A}_{t} \varphi_{k}\right)\right\}_{k \geq 1}$ is dense in $\left\{\left(\varphi, \mathcal{A}_{t} \varphi\right), \varphi \in C_{b}^{2}\right\}$ for the bounded-pointwise convergence;

(ii) for any $\left(t_{0}, x_{0}\right)$ in $[0, T] \times \mathbb{R}^{3}$, there exists a unique (in law) solution $\left(X_{t}\right)_{t \in\left[t_{0}, T\right]}$ to the martingale problem for $\left(C_{b}^{2}, \mathcal{A}_{t}\right)$ such that $X_{t_{0}}=x_{0}$.

We now verify these two points. First consider a countable family of functions $\left(\varphi_{k}\right)_{k \geq 1} \subset C_{b}^{2}$, dense in $C_{b}^{2}$ for the norm $\|\varphi \varphi\|:=\|\varphi\|_{\infty}+\|D \varphi\|_{\infty}+\left\|D^{2} \varphi\right\|_{\infty}$. Then point (i) easily follows (with the uniform convergence instead of the bounded-pointwise convergence) from the estimate $\left|\mathcal{A}_{t} \varphi(x)\right| \leq \int_{\mathbb{R}^{3}}|L \varphi(x, v)| f_{t}(v) d v \leq C|||\varphi||| \int_{\mathbb{R}^{3}}\left(|x-v|^{-1}+|x-v|^{-2}\right) f_{t}(v) d v \leq C|\| \varphi|||\left(1+\left\|f_{t}\right\|_{\infty}\right)$ due to (7) with $\alpha=-1$ and $\alpha=-2$. To prove (ii), observe that the martingale problem for $\left(C_{b}^{2}, \mathcal{A}_{t}\right)$ with $X_{t_{0}}=x_{0}$ corresponds to the stochastic differential equation

$$
X_{t}=x_{0}+\int_{t_{0}}^{t} \int_{\mathbb{R}^{3} \times \mathbb{R}^{3}} \sigma\left(X_{s}-v\right) W(d v, d \tilde{v}, d s)+\int_{t_{0}}^{t} \int_{\mathbb{R}^{3}} b\left(X_{s}-v\right) f_{s}(d v) d s,
$$

for which we have shown the strong existence and uniqueness (only in the case $t_{0}=0$ and $x_{0}=V_{0}$, but the generalization is straightforward). Point (ii) follows.

\section{CONCLUSiOn}

Our main result is easily deduced from Theorem 9 and Lemma 8 .

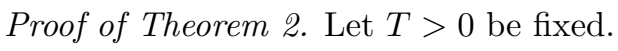

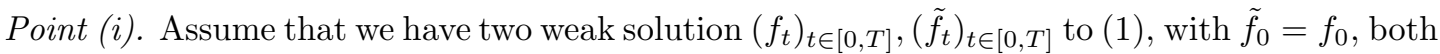
belonging to $L^{\infty}\left([0, T], \mathcal{P}_{2}\right) \cap L^{1}\left([0, T], L^{\infty}\right)$. Theorem 9 implies that there is a bounded function $\rho:[0, T] \mapsto \mathbb{R}_{+}$such that $\mathcal{W}_{2}^{2}\left(f_{t}, \tilde{f}_{t}\right) \leq \rho(t)$ for all $t \in[0, T]$ satisfying $\rho(t) \leq \int_{0}^{t} \gamma(s) \Psi(\rho(s)) d s$, with $\gamma(s)=C\left(1+\left\|f_{s}+\tilde{f}_{s}\right\|_{\infty}\right) \in L^{1}([0, T])$. Lemma 8 (i) implies that $\rho(t)=0$, whence $\mathcal{W}_{2}\left(f_{t}, \tilde{f}_{t}\right)=0$, for all $t \in[0, T]$. Thus $\left(f_{t}\right)_{t \in[0, T]}=\left(\tilde{f}_{t}\right)_{t \in[0, T]}$.

Point (ii). Let now $\left(f_{t}\right)_{t \in[0, T]},\left(f_{t}^{n}\right)_{t \in[0, T]}$ be a family of weak solutions such that $R_{T}:=$ $\sup _{n} \int_{0}^{T}\left(1+\left\|f_{s}^{n}+f_{s}\right\|_{\infty}\right) d s<\infty$ and $a_{n}:=\mathcal{W}_{2}^{2}\left(f_{0}^{n}, f_{0}\right) \rightarrow 0$. Applying Theorem 9 we get $\mathcal{W}_{2}^{2}\left(f_{t}^{n}, f_{t}\right) \leq \rho_{n}(t)$, for some bounded function $\rho_{n}:[0, T] \mapsto \mathbb{R}_{+}$satisfying

$$
\rho_{n}(t) \leq a_{n}+\int_{0}^{t} C\left(1+\left\|f_{s}^{n}+f_{s}\right\|_{\infty}\right) \Psi\left(\rho_{n}(s)\right) d s .
$$


Lemma 8 -(ii) implies $M\left(a_{n}\right)-M\left(\rho_{n}(t)\right) \leq C R_{T}$, where $M(x)=\int_{x}^{1}(1 / \Psi(y)) d y$ is decreasing on $(0, \infty)$ and satisfies $\lim _{x \searrow 0} M(x)=+\infty$. As a consequence,

$$
\liminf _{n} M\left(\sup _{[0, T]} \rho_{n}(t)\right)=\liminf _{n} \inf _{[0, T]} M\left(\rho_{n}(t)\right) \geq \liminf _{n} M\left(a_{n}\right)-C R_{T}=+\infty .
$$

This implies that $\lim _{n} \sup _{[0, T]} \rho_{n}(t)=0$, and finally, $\lim _{n} \sup _{[0, T]} \mathcal{W}_{2}^{2}\left(f_{t}^{n}, f_{t}\right)=0$.

\section{REFERENCES}

[1] R. Alexandre, C. Villani, On the Landau approximation in plasma physics, Ann. Inst. H. Poincaré Anal. Non Linéaire 21 (2004), 61-95.

[2] A.A Arsen'ev, N.V. Peskov, The existence of a generalized solution of Landau's equation, Z. Vycisl. Mat. i Mat. Fiz. 17 (1977), 1063-1068.

[3] A.G. Bhatt, R.L. Karandikar, Invariant measures and evolution equations for Markov processes characterized via martingale problems, Ann. Probab. 21 (1993), 2246-2268.

[4] J.Y. Chemin, Fluides parfaits incompressibles, Astérisque No. 230, 1995.

[5] L. Desvillettes, C. Villani, On the spatially homogeneous Landau equation for hard potentials, Part I : existence, uniqueness and smothness, Comm. Partial Differential Equations 25 (2000), 179-259.

[6] N. Fournier, C. Mounot, On the well-posedness of the spatially homogeneous Boltzmann equation with a moderate angular singularity, Comm. Math. Phys. 289 (2009), 803-824.

[7] N. Fournier, H. Guérin, On the uniqueness for the spatially homogeneous Boltzmann equation with a strong angular singularity, Journal of Statistical Physics 131 (2008), 749-781.

[8] N. Fournier, H. GuÉrin, Well-posedness of the spatially homogeneous Landau equation for soft potentials, J. Funct. Anal. 256 (2009), 2542-2560.

[9] T. FunAKI, The diffusion approximation of the spatially homogeneous Boltzmann equation, Duke Math. J. 52 (1985), 1-23.

[10] H. GuÉRIN, Solving Landau equation for some soft potentials through a probabilistic approach, Ann. Appl. Probab. 13 (2003), 515-539.

[11] J. Horowitz, R.L. Karandikar, Martingale problems associated with the Boltzmann equation, Seminar on Stochastic Processes, 1989 (San Diego, CA, 1989), 75122, Progr. Probab., 18, Birkhauser Boston, Boston, MA, 1990.

[12] H. Tanaka, Probabilistic treatment of the Boltzmann equation of Maxwellian molecules, Z. Wahrsch. und Verw. Gebiete 46 (1978/79), 67-105.

[13] C. Villani, On the spatially homogeneous Landau equation for Maxwellian molecules., Math. Models Methods Appl. Sci. 8 (1998), 957-983.

[14] C. Villani, On a new class of weak solutions to the spatially homogeneous Boltzmann and Landau equations, Arch. Rational Mech. Anal. 143 (1998), 273-307.

[15] C. Villani, A review of mathematical topics in collisional kinetic theory, Handbook of mathematical fluid dynamics, Vol. I, 71-305, North-Holland, Amsterdam, 2002.

[16] C. Villani, Topics in Optimal Transportation, Graduate Studies in Mathematics Vol. 58, AMS 2003.

[17] J.B. WALSH, An introduction to stochastic partial differential equations, École d'été de Probabilités de SaintFlour XIV, Lect. Notes in Math. 1180, 265-437, 1986.

LAma UMR 8050, Faculté de Sciences et Technologies, Université Paris Est, 61, avenue du Général de Gaulle, 94010 Créteil Cedex, France

E-mail address: nicolas.fournier@univ-paris12.fr 